\title{
STOKES FLOW IN A RECTANGULAR WELL: NATURAL CONVECTION AND BOUNDARY-LAYER FUNCTION*
}

BY

L. M. DE SOCIO (Polytechnic of Turin)

G. GAFFURI AND L. MISICI (University of Camerino)

\begin{abstract}
Free convection in a rectangular well of infinite depth at low Rayleigh number is considered. A pair of opposite vertical walls are at different temperatures, whereas the other walls are adiabatic. The three-dimensional Stokes flow regime is analyzed and the boundary-layer function for the adiabatic walls is determined.
\end{abstract}

1. Introduction. Problems of natural convection in enclosures have received renewed and increasing attention in the last decade. This is mainly due to engineering and physical applications, as, for example, the slow free convection in energy storage systems, solar collectors, glass melting tanks, and pollution in basins.

From the point of view of applied mathematics, the problem of natural convection taking place at low Rayleigh number in liquid-filled two-dimensional cavities and trenches of different cross-sectional shapes has been analytically solved in a number of papers, following the pioneering paper of Joseph and Sturges [1]. In all cases, a Stokes flow results where, for sufficiently low Rayleigh number, conduction is the basic mode of heat transfer between two walls at different temperatures [2].

In this paper, an analytical solution is obtained for the flow field in a well of rectangular cross-section and of infinite depth, in the presence of a free surface, when two vertical opposite walls are kept at different temperatures and the other two walls are adiabatic.

After determining the solution in this situation, an interesting singular perturbation problem arises, i.e. to express the solution of the present three-dimensional configuration, as the distance between the adiabatic walls tends to infinity, in terms of the solution to the resulting two-dimensional problem and of a boundary-layer function.

In the following, it is assumed that the viscosity and the heat conductivity of the medium above the free surface can be neglected in comparison with the analogous quantities of the liquid in the cavity. The Rayleigh number being small, the free surface will, in this circumstance, deviate only negligibly from the horizontal plane.

2. Basic equations. With reference to Fig. 1, a system of Cartesian axes is considered, with the $z=0$ plane containing the free surface and the $z$-axis pointing positively upward. All the distances are made non-dimensional with respect to the semi-width of the well, $1 / 2 a$.

For the present problem it is easy to show that the velocity component along $y$ vanishes

* Received October 15, 1980. This work was partially supported by the Italian National Research Council through CNR Contract No. 79.02817.11. 


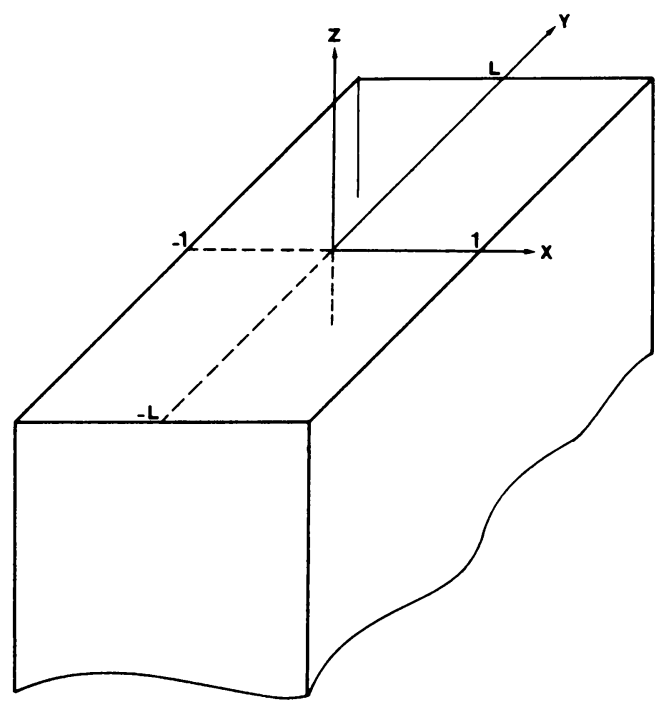

FIG. 1. A sketch of the cavity.

everywhere. Therefore, a stream function $\Psi$ can be introduced and the set of basic equations in the Oberbeck-Boussinesq approximation is given by [3]:

$$
\begin{gathered}
\Delta_{3} \theta=0, \\
\mathscr{L}[\Psi] \equiv \Delta_{2}^{2} \Psi+\Delta_{2} \Psi_{y y}=\theta_{x},
\end{gathered}
$$

where $\Psi$ has been made dimensionless with respect to $\rho g \alpha a^{3} / 16 \mu$, with $\rho, \alpha$, and $\mu$ representing the density, thermal expansion coefficient, and viscosity of the fluid, respectively, and with $g$ the acceleration of gravity. In Eq. (1), $\theta=\left(T-T_{-1}\right) /\left(T_{1}-T_{-1}\right)$, where $T$ is the temperature and the indices -1 and 1 refer to the walls at $x=-1$ and $x=1$ respectively. Furthermore, $\Delta_{3}=()_{x x}+()_{y y}+()_{z z}$ and $\Delta_{2}=()_{x x}+()_{z z}$.

The pertinent boundary conditions are

$$
\begin{aligned}
\theta(-1, y, z)=0 ; \theta(1, y, z)=1 ; \theta_{y}(x,-L, z)=\theta_{y}(x, L, z) & =\theta_{z}(x, y, 0) \\
& =\theta_{z}(x, y,-\infty)=0
\end{aligned}
$$

and

$$
\Psi( \pm 1, y, z)=\Psi_{x}( \pm 1, y, z)=\Psi_{x}(x, y,-\infty)=\Psi(x, y, 0)=\Psi_{z z}(x, y, 0)=0
$$

and $\Psi(x, y,-\infty)$ bounded,

$$
\Psi(x, \pm L, z)=0 \text {. }
$$

Since the solution of Eq. 1 subject to the conditions (3) is simply

$$
\theta=x+1 / 2
$$

Eq. (2) can be written as

$$
\mathscr{L}[\Psi]=1
$$

with the associated conditions (4), and the stream function can be expanded into the 
Fourier series

$$
\Psi(x, y, z)=\sum_{j=0}^{\infty} \Psi_{j}(x, z) \cos \left(\lambda_{j} \delta y\right)
$$

where

$$
\lambda_{j} \delta=\pi(2 j+1) / 2 L
$$

and $\delta=L^{-1}, \lambda_{j}=\pi(2 j+1) / 2$.

Expression (7) satisfies the conditions (4b) and can be introduced into Eq. (6), so that

$$
\sum_{j=0}^{\infty} \cos \left(\lambda_{j} \delta y\right) \mathscr{L}_{j}\left[\Psi_{j}\right]=1
$$

with $\mathscr{L}_{j}[\cdot] \equiv \Delta_{2}^{2}(\cdot)-\lambda_{j}^{2} \delta^{2} \Delta(\cdot)$.

Standard operations lead to the equations for the $\Psi_{j}$

$$
\mathscr{L}_{j}\left[\Psi_{j}\right]=(-1)^{j} 2 / \lambda_{j}, \quad(j=0,1,2 \ldots)
$$

subject to

$$
\begin{aligned}
\Psi_{j}=( \pm 1, z)=\Psi_{j, x}( \pm 1, z)=\Psi_{j}(x,-\infty) & \\
& =\Psi_{j, z}(x,-\infty)=\Psi_{j}(x, 0)=\Psi_{j, z z}(x, 0)=0
\end{aligned}
$$

3. Solution. The solution to Eqs. (10) can be expressed in the form

$$
\Psi_{j}(x, z)=\Psi_{P j}(x)+\Psi_{H j}(x, z)
$$

where the particular integral $\Psi_{P j}$ is given by

$$
\begin{aligned}
\Psi_{P j}(x)= & {\left[(-1)^{j} / \lambda_{j}^{3} \delta^{2}\right]\left[-x^{2}+2 \cosh \lambda_{j} \delta x+\lambda_{j} \delta \sinh \lambda_{j} \delta\right.} \\
& \left.\left.-2 \cosh \lambda_{j} \delta\right) /\left(\lambda_{j} \delta \sinh \lambda_{j} \delta\right)\right]
\end{aligned}
$$

and $\Psi_{H j}(x, z)$ is the general integral of the homogeneous equation

$$
\Delta^{2} \Psi_{H j}-\lambda_{j}^{2} \delta^{2} \Delta \Psi_{H j}=0
$$

with

$$
\begin{aligned}
\Psi_{H j}( \pm 1, z) & =\Psi_{H j, x}( \pm 1, z)=\Psi_{H j, z z}(x, 0)=\Psi_{H j}(x,-\infty)=\Psi_{H j, z}(x,-\infty)=0, \\
\Psi_{H j}(x, 0) & =-\Psi_{P j}(x) .
\end{aligned}
$$

Following Smith's approach [4] as in [1], one can evaluate the solution to Eq. (14) in the form of a Papkovich-Fadle series

$$
\Psi_{H j}(x, z)=C_{j}^{(n)} \exp \left[s_{j}^{(n)} z\right] \Phi_{1 j}^{(n)}(x) / s_{j}^{(n) 2}
$$

where

$$
\Phi_{1 j}^{(n)}=s_{j}^{(n) 2}\left[\cos \mu_{j}^{(n)} \cos \left(s_{j}^{(n)} x\right)-\cos s_{j}^{(n)} \cos \left(\mu_{j}^{(n)} x\right)\right]
$$

with

$$
\mu_{j}^{(n)}=\left(s_{j}^{(n) 2}-\lambda_{j}^{2} \delta^{2}\right)^{1 / 2}
$$


In order to satisfy the boundary conditions at the lateral walls, the $s_{j}^{(n)}$ are the solutions of the transcendental equation

$$
[\sin (s+\mu)] /(s+\mu)=-[\sin (s-\mu)] /(s-\mu)
$$

where $\mu=\left(s^{2}-\lambda \delta\right)^{1 / 2}$.

The coefficients $C_{j}^{(n)}$ are evaluated by imposing the conditions at the free surface and their expression is given by

$$
C_{j}^{(n)}=W_{j}^{(n)} / K_{j}^{(n)}
$$

with

$$
\begin{aligned}
W_{j}^{(n)} & =\int_{-1}^{1}\left[\phi_{1 j}^{(n)}, \phi_{2 j}^{(n)}\right] \cdot \mathbf{A}\left[\begin{array}{c}
0 \\
-\Psi_{P, x x}
\end{array}\right] d x, \\
K_{j}^{(n)} & =\int_{-1}^{1}\left[\phi_{1 j}^{(n)}, \phi_{2 j}^{(n)}\right] \cdot \mathbf{A}\left[\begin{array}{c}
\Phi_{1 j}^{(n)} \\
\Phi_{2 j}^{(n)}
\end{array}\right] d x,
\end{aligned}
$$

and

$$
\begin{gathered}
\mathbf{A}=\left[\begin{array}{rr}
0 & -1 \\
1 & 2
\end{array}\right], \\
\Phi_{2 j}^{(n)}(x)=-s_{j}^{(n) 2} \cos \mu_{j}^{(n)} \cos \left(s_{j}^{(n)} x\right)+\mu_{j}^{(n) 2} \cos s_{j}^{(n)} \cos \left(\mu_{j}^{(n)} x\right), \\
\phi_{1 j}^{(n)}(x)=\mu_{j}^{(n) 2} \cos \mu_{j}^{(n)} \cos \left(s_{j}^{(n)} x\right)-s_{j}^{(n) 2} \cos s_{j}^{(n)} \cos \left(\mu_{j}^{(n)} x\right), \\
\phi_{2 j}^{(n)}(x)=\Phi_{1 j}^{(n)}(x) .
\end{gathered}
$$

Omitting the lengthy but straightforward algebra, one has

$$
K_{j}^{(n)}=\lambda_{j}^{2} \delta^{2}\left[s_{j}^{(n) 2}\left(\cos ^{2} s_{j}^{(n)}-\cos ^{2} \mu_{j}^{(n)}\right)+\left(\lambda_{j}^{2} \delta^{2} s_{j}^{(n)} / \mu_{j}^{(n) 2}\right) \sin s_{j}^{(n)} \cos s_{j}^{(n)} \cos ^{2} \mu_{j}^{(n)}\right]
$$

and

$$
W_{j}^{(n)}=(-1)^{j}\left(-4 \lambda_{j} \delta^{2} / s_{j}^{(n)} \mu_{j}^{(n) 2}\right) \sin s_{j}^{(n)} \cos \mu_{j}^{(n)} .
$$

Remark 1. The solution (16) is bounded for any $y \in(0,-\infty)$, provided that the roots $s_{j}^{(n)}$ of Eq. (18) are the real positive and the complex conjugate roots in the first and fourth quadrants. The number of real positive $s_{j}^{(n)}$ depends upon $\Gamma_{j}=\lambda_{j} \delta$ and, in particular, there exists a denumerable infinity $\left\{\Gamma_{j}^{*}\right\}$ of values of $\Gamma_{j}$ for which Eq. (18) admits a pair of real coincident roots, $\gamma_{j}^{(i)}$. As an indication one can verify that:

$\begin{array}{cc}\gamma_{j}^{(i)} & \Gamma_{j}^{*} \\ 2.7984 & 3.0447 \\ 6 \cdot 1213 & 6 \cdot 2377 \\ 9 \cdot 3179 & 9 \cdot 3948\end{array}$

On the basis of Remark 1, the solution (16) can be put into the form

$$
\Psi_{H j}(x, z)=\sum_{n=1}^{\infty} a_{j}^{(n)} \mathscr{R}\left[C_{j}^{(n)} \exp \left\{s_{j}^{(n)} z\right\} \Phi_{1 j}^{(n)}(x) / s_{j}^{(n) 2}\right]
$$

where $a_{j}^{(n)}=1$, for $\mathscr{J}\left[s_{j}^{(n)}\right]=0 ; a_{j}^{(n)}=2$ for $\mathscr{J}\left[s_{j}^{(n)}\right] \neq 0$. 
Remark 2. From Eq. (22) it is easy to see that

for any positive integer $j$.

$$
\lim _{z \rightarrow-\infty} \Psi_{H j}(x, z)=0
$$

4. The flow field. The distribution of iso- $\Psi$ lines at some selected $y=$ const planes has been evaluated in a number of cases. Figs. $2 \mathrm{a}$ and $2 \mathrm{~b}$ correspond to the values $L=0.2$ and $L=1$ (square cross-section). In each case, as expected, the maximum value of the stream function in each plane $y=$ const decreases regularly with increasing $y$. Furthermore, if $\Psi_{\text {max }, 0}$ denotes the maximum of $\Psi$ in the plane $y=0$, this value decreases with $L$, as can be easily realized from a physical point of view.

Fig. 3 shows the behavior of $\Psi_{\max , 0}$ versus $L$. For $L=0, \Psi_{\max , 0}=0$, whereas, as $L \rightarrow \infty, \Psi_{\max , 0}$ tends to the maximum value of the stream function for the trench of infinite length $(2 / 31$ as in $[1])$.

The convergence of the Fourier series (7) and of the Papkovich-Fadle series (22) proved to be faster at smaller $L$ for a given truncation error of $10^{-4}$. The analytical solution presented here can, if required, immediately allow the evaluation of the vorticity distribution in the field as, for example, in the estimate of the initial guess for the iterative solution of the full Oberbeck-Boussinesq equations (for the plane case, this procedure was followed in [5]).

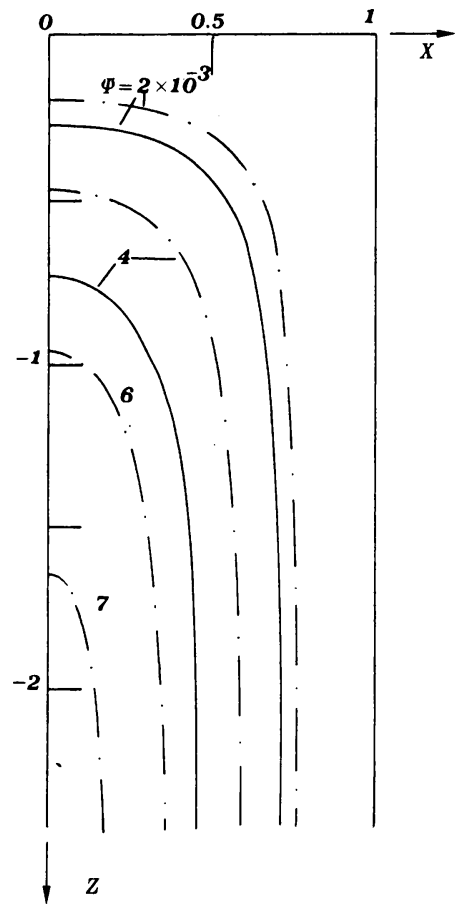

Fig. 2a. Iso- $\Psi$ lines at $y=0$ (broken lines) and $y=0.1$ (solid lines), for $L=0.2$.

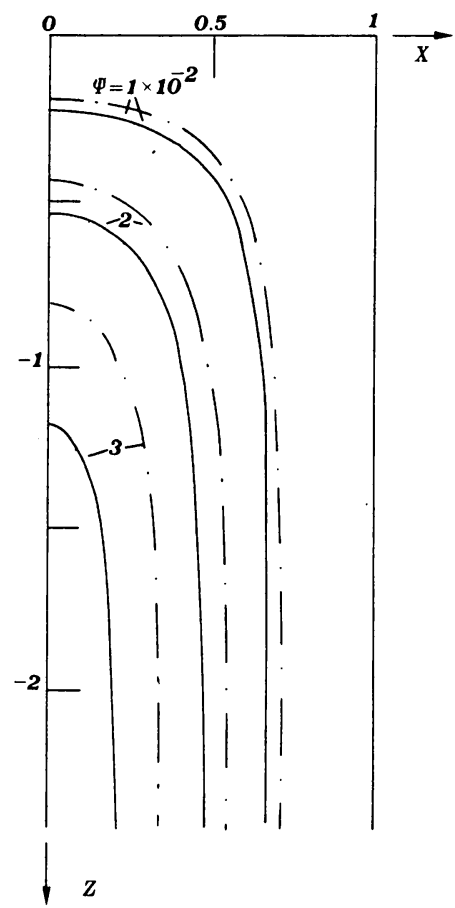

FIG. 2b. Iso- $\Psi$ lines at $y=0$ (broken lines) and $y=0.5$ (solid lines), for $L=1$. 


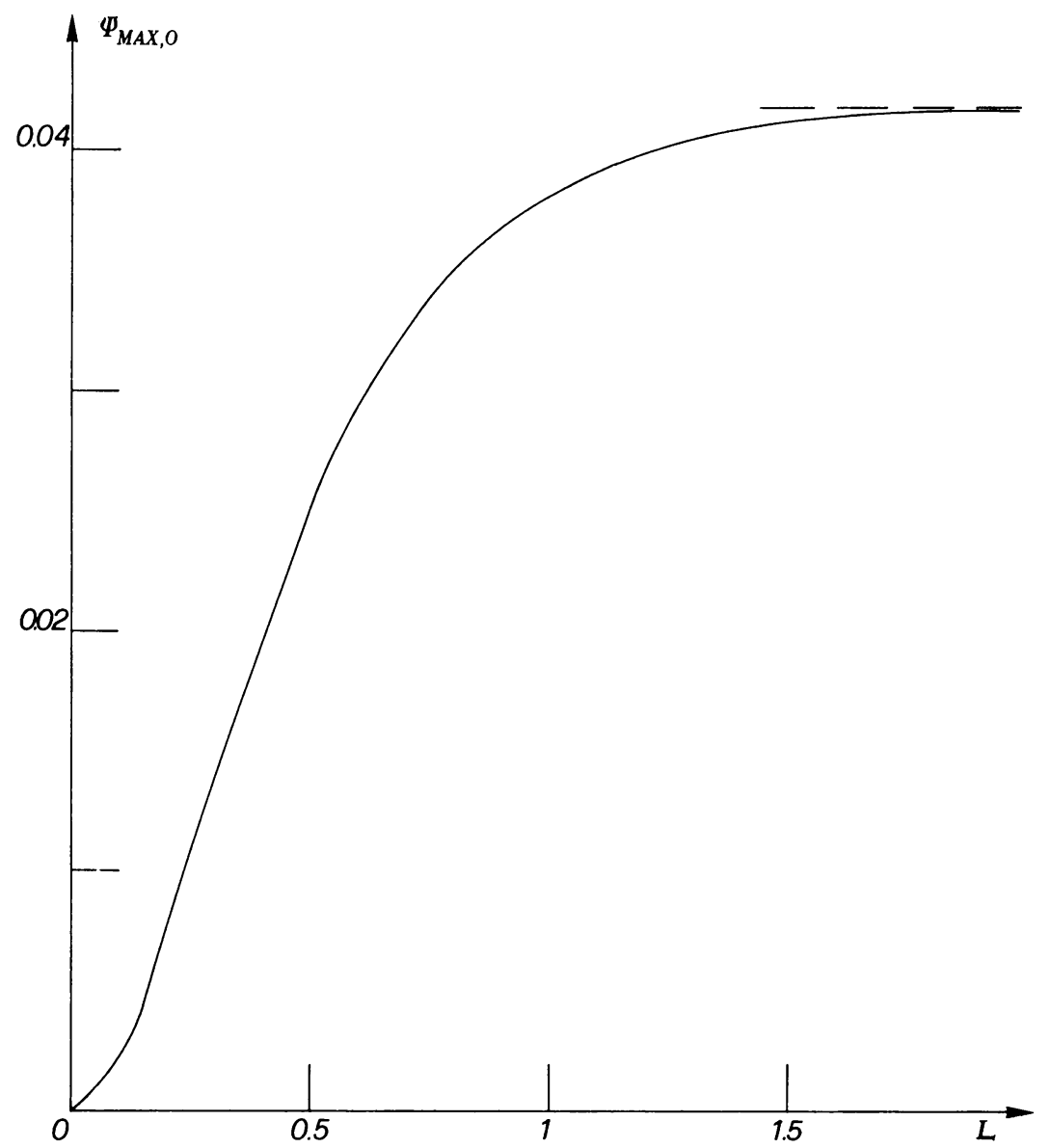

FIG. 3. The behavior of $\Psi_{\max , 0}$ versus $L$. The dashed line corresponds to the maximum value of the stream function for the two-dimensional trench of infinite length.

5. The singular perturbation problem. The operator $\mathscr{L}[\Psi(x, y, z)]$, after introducing the new variable $t=1-y / L$, can be written

$$
\mathscr{L}[\Psi(x, y, z)]=\mathscr{L}^{*}\left[\Psi^{*}(x, t, z)\right]=1
$$

where $\mathscr{L}^{*} \equiv \Delta_{2}^{2}+\delta^{2} \Delta_{2}\left(\partial^{2} / \partial t^{2}\right)$. The boundary conditions for (23) are easily obtained from (4.a, b).

A classical problem of small parameter arises from Eq. (23) as $\delta \rightarrow 0$. In principle one might think of expressing $\psi^{*}$, which is known at this point, as a function of the solution of the biharmonic operator, obtained from $\mathscr{L}^{*}$ by putting $\delta=0$, and of a boundary layer function plus a function representing the error. However, the solution to the biharmonic equation for the rectangular domain considered, cannot be obtained explicitly by variable separation [7].

As the solution to the biharmonic equation cannot be expressed in terms of elementary functions, it would be cumbersome to face the boundary-layer problem in its generality, 


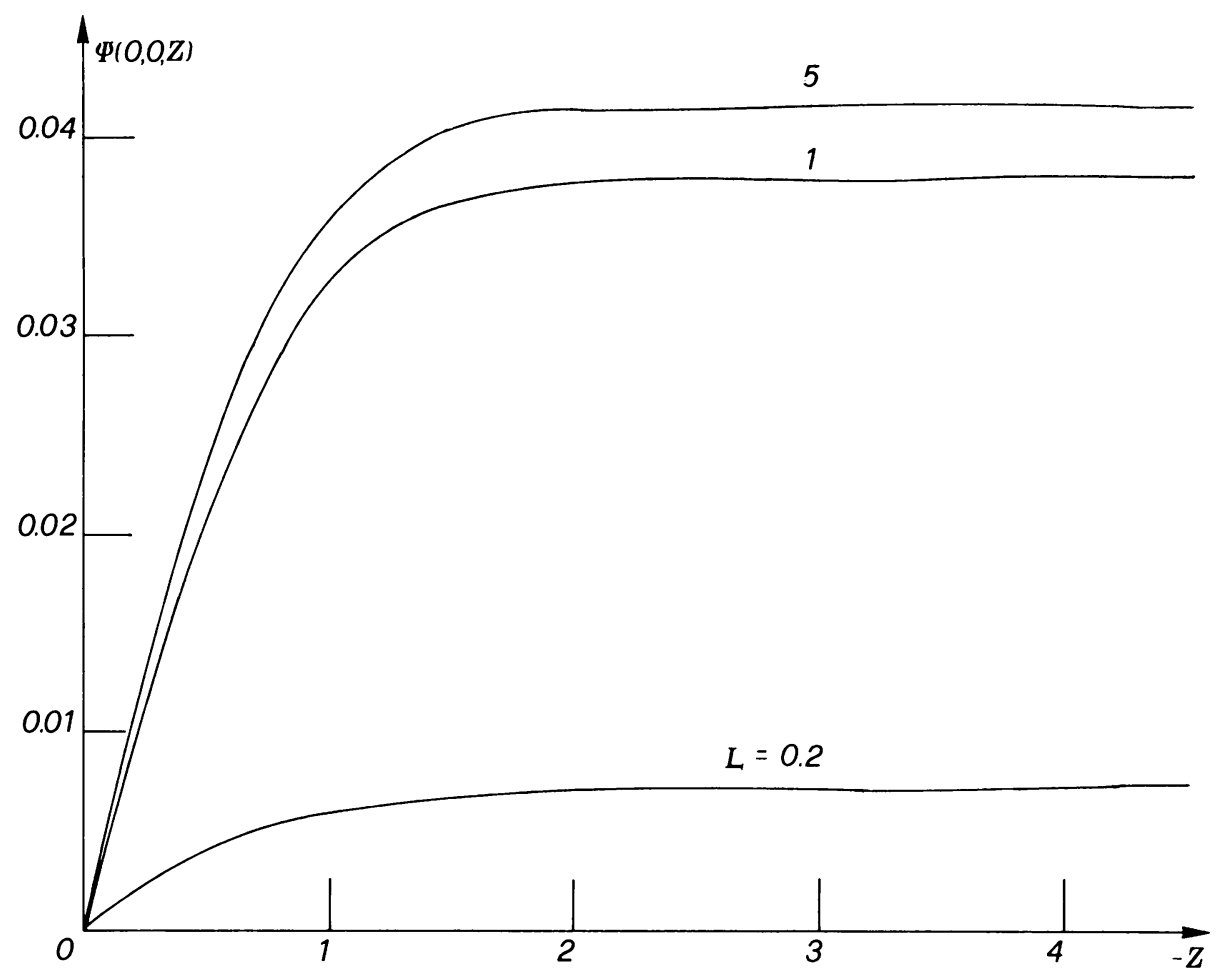

FIG. 4. The solution along the symmetry axis $\Psi(0,0, z)$ at various $L$.

and the lengthy algorithm, which is straightforward in principle, would obscure the physical meaning of the procedure.

In order to make the problem more tractable without loss of generality, the following considerations can be discussed. When the behavior of the solution (7) is taken into account at increasing depth $z$, the contribution given by the terms containing the Papkovich-Fadle series becomes rapidly negligible (in this respect one should recall Remark 2). To this purpose one can observe Fig. 4, showing how the solution evaluated in the symmetry plane of the trench, $x=0$, at $y=0$ varies with $z$. It can be realized that, at different $L$, the particular integral can be considered instead of the general solution, with relative errors rapidly decreasing at increasing $|z|$. This is even more evident when the solution at high values of $x$ and $y$ is evaluated.

Therefore, our goal is to show that the solution of Eq. (23) at infinite depth $z=-\infty$, i.e. $\phi(x, t)=\Psi^{*}(x, t,-\infty)$, can be written as the sum of the analogous solution of the biharmonic problem at $z=-\infty$ and of a boundary-layer function, plus a function representing the error made when $\phi(x, t)$ is represented by the only first two terms of the sum.

The equation for $\phi(x, t)$ is

$$
\frac{\partial^{4} \phi}{\partial x^{4}}+\delta^{2} \frac{\partial^{4} \phi}{\partial x^{2} \delta t^{2}}=1, \quad t \in(0,1)
$$


with

$$
\phi( \pm 1, t)=\left.\frac{\partial \phi}{\partial x}\right|_{( \pm 1, t)}=0, \quad \phi(x, 0)=0 ;\left.\quad \frac{\partial \phi}{\partial t}\right|_{(x, 1)}=0 .
$$

When the technique of matched composite asymptotic expansion (MCAE) is adopted [6], a first approximation of the solution to Eqs. $(24,25)$ can be written in the form

$$
\phi_{0}(x, t, \delta)=\frac{\left(1-x^{2}\right)^{2}}{24}+2 \sum_{k=1}^{\infty}(-1)^{k} \frac{\cos k \pi x-1}{k^{4} \pi^{4}} \exp (-k \pi t / \delta)
$$

Note that $\phi_{0}(x, t, \delta) \rightarrow \phi(x, t)$ as $\delta \rightarrow 0$, uniformly. Furthermore, from Eq. (26) and Eqs. (12, $13,16)$ one can prove that

$$
\sup _{\substack{-1 \leq x \leq 1 \\ 0 \leq t \leq 1}}\left|\phi(x, t)-\phi_{0}(x, t, \delta)\right| \leq \frac{4}{\pi^{4}} \exp (-\pi / \delta)=E
$$

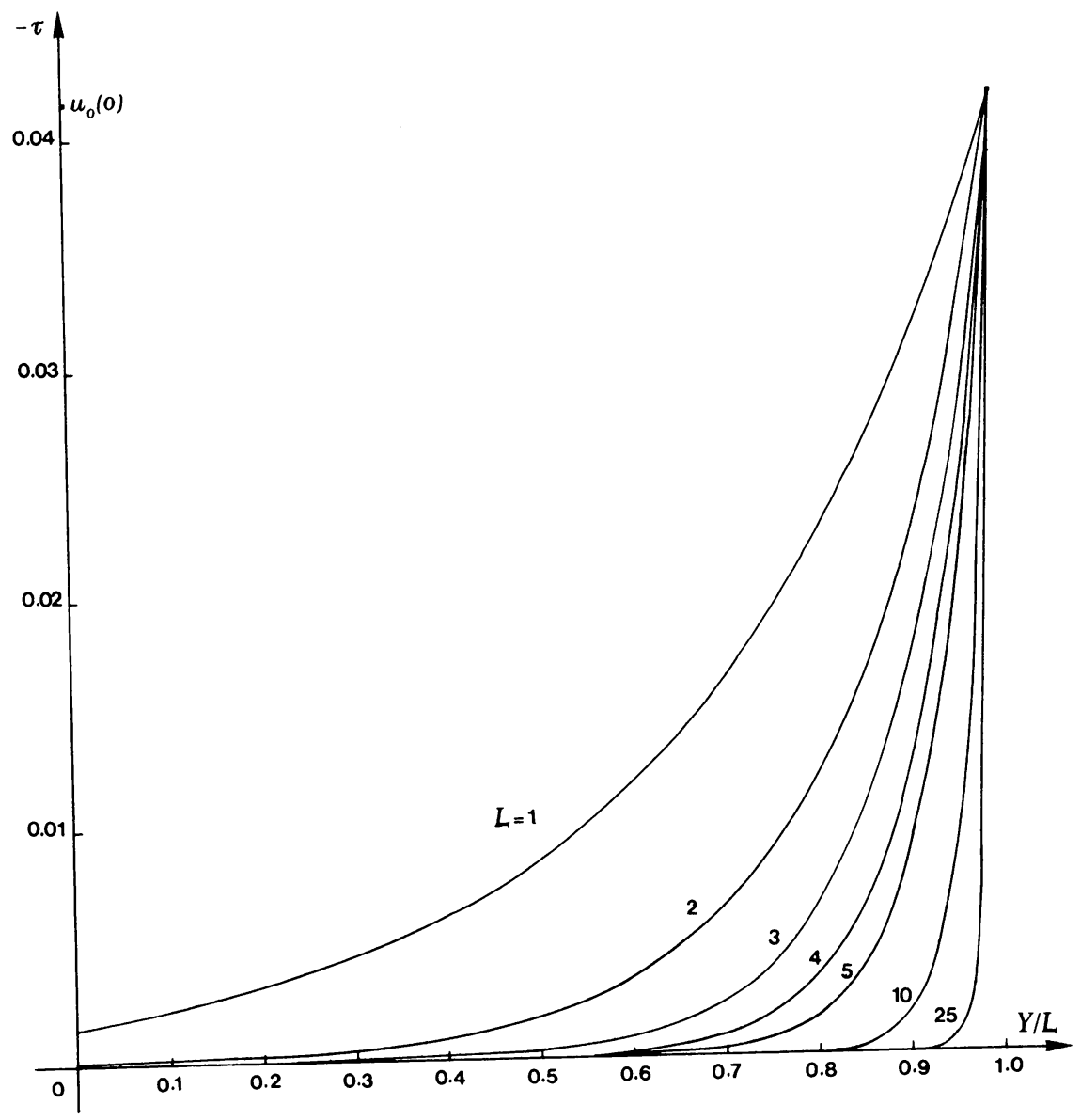

FIG. 5. The boundary layer function $\tau$ versus the normalized coordinate $y / L$, for various $L$, at $x=0$.

$$
u_{0}(0)=0.0417
$$




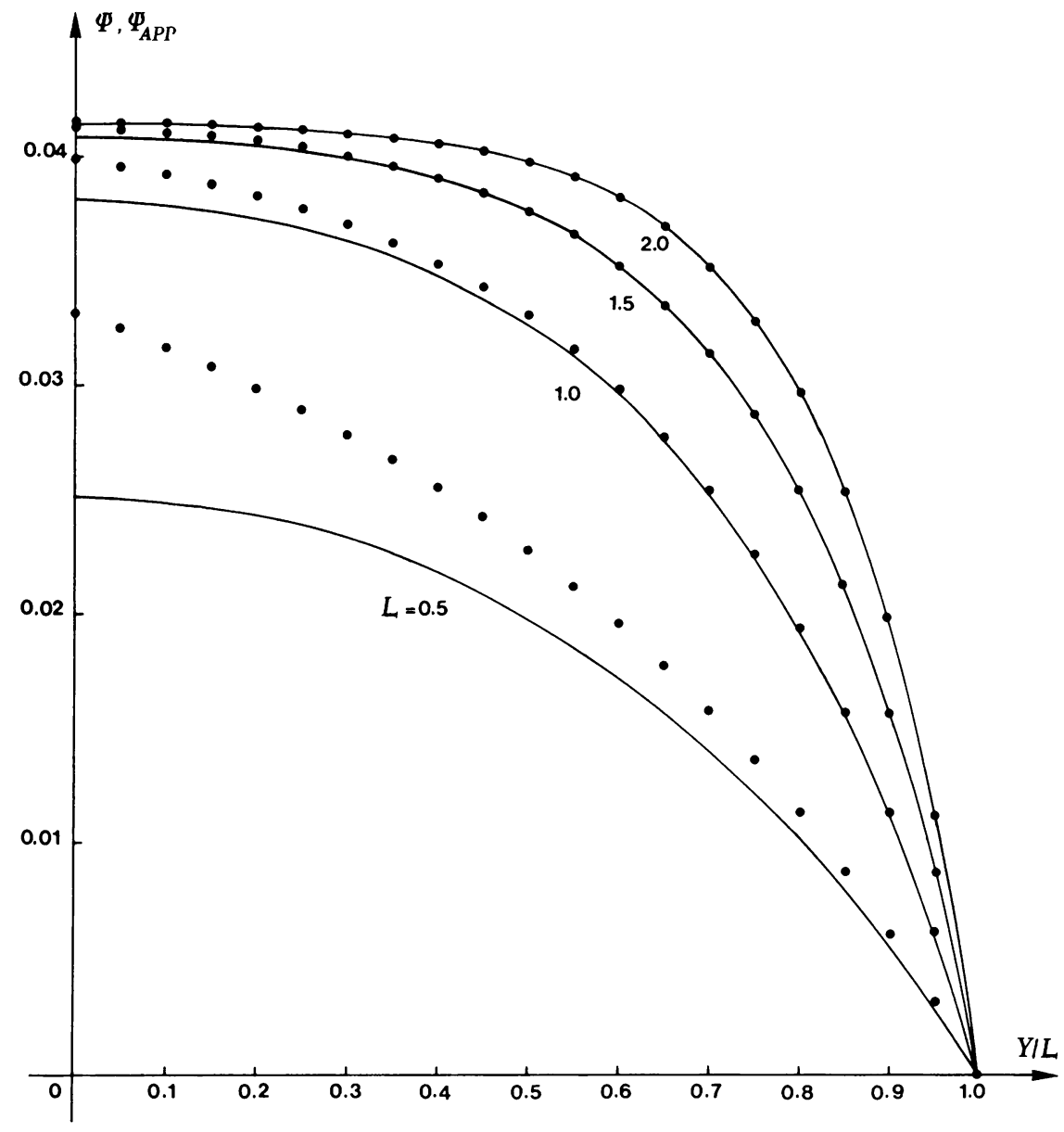

Fig. 6. The true solution $\Psi$ (solid lines) and the composite asymptotic solution $\Psi_{\text {APP }}$ (dots) versus the normalized coordinate $y / L$ for various $L$, at $x=0$.

where $E$ is the maximum error. Therefore, for sufficiently high $|z|$, one can put

$$
\psi^{*}(x, t,-\infty)=u_{0}(x)+\tau(x, y, \delta)+E
$$

where $u_{0}(x)$ is the solution for the problem of the trench of infinite length, $\left(1-x^{2}\right) / 24$, whereas $\tau(x, y, \delta)$ is a boundary-layer function such that, for $y= \pm L$

$$
\tau(x, \pm L, \delta)=-u_{0}(x)
$$

and

$$
\tau(x, y, \delta)=2 \sum_{k=1}^{\infty}(-1)^{k} \frac{\cos k \pi x-1}{k^{4} \pi^{4}} \exp \{-k \pi(1-|y| \delta) / \delta\} .
$$

Fig. 5 shows the behavior of the boundary-layer function versus $|y| / L$ at several $L=\delta^{-1}$ and for $x=0$. Fig. 6 gives the exact solution and the approximate solution $\Psi_{\mathrm{APP}}$, 
(26) at $x=0$ for different $L$. As one can see, the comparison between the two solutions is excellent even for values of $L$ a little higher than unity. For $x>0$, numerical computations show that the agreement improves, as can be easily understood.

Expression (29) clearly indicates how the boundary-layer function, as $\delta \rightarrow 0$, contributes negligibly everywhere to the solution, with the exception of the narrow regions close to the walls, where $y$ is of the order of magnitude $\delta^{-1}$. In any case, the order of magnitude of the maximum error is a function of $\delta$ and is given through Eq. (27). On the other hand, the thickness of the boundary layer exponentially decreases with $\delta$, as shown by expression (29) and Fig. 5.

6. Conclusion. In this work the problem of the Stokes flow taking place in a rectangular well of infinite depth under the action of buoyancy forces has been analyzed. The three-dimensional solution has been analytically given in terms of a Papkovich-Fadle series and the characteristics of the flow field have been discussed. The singular perturbation problem arising when the length of the well becomes infinite has been approached by means of the matched composite asymptotic expansion, and the properties of the boundary-layer function and boundary-layer thickness have been considered.

\section{REFERENCES}

[1] D. D. Joseph and L. Sturges, The free surface on a liquid filling a trench heated from its side, J. Fluid Mech. 69, 565-589(1975)

[2] E. R. G. Eckert and R. M. Drake, Jr., Analysis of heat and mass transfer, McGraw-Hill, New York, 1972

[3] D. D. Joseph, Stability of fluid motions, Vol. 2, Springer-Verlag, Berlin, 1976

[4] R. C. T. Smith, The bending of a semi-infinite strip, Aust. J. Sci. Res. 5, 227-237 (1952)

[5] L. M. de Socio, L. Misici and A. Polzonetti, Natural convection in heat generating fluids in cavities, ASME Paper 79-HT-95

[6] P. A. Lagerström and R. G. Casten, Basic concepts underlying singular perturbation techniques, SIAM Review 14, 63-120(1972)

[7] R. Courant and R. Hilbert, Methods of mathematical physics, Vol. 1, Interscience Publishers, New York, 1953 